\title{
UNA APROXIMACIÓN PRAXEOLÓGICA A LA ENERGÍA
}

\author{
ANTONIO ESPAÑA CONTRERAS*
}

\begin{abstract}
Resumen: El presente artículo analiza el concepto físico y práctico de energía desde la perspectiva de la acción humana o praxeológica, aplicando en esta tarea el instrumental analítico desarrollado por la escuela austriaca de economía. Para hacer comprensible el análisis, el trabajo desarrolla con anterioridad el concepto de la energía desde el punto de vista ontológico y científico, así como ofrece un breve bosquejo histórico de su utilización por el ser humano para lograr sus metas. Una vez sentadas las bases para un entendimiento común, el artículo justifica la consideración de la energía como el bien económico de orden superior por excelencia, demuestra la importancia de la eficiencia dinámica en su estudio, muestra su estrecha relación con la riqueza de las naciones y la importancia de su papel en las etapas del proceso productivo.
\end{abstract}

Palabras clave: Energía y economía, energía y praxeología, historia del concepto de energía, historia de la aplicación práctica de la energía, energía como bien económico, energía y riqueza.

Clasificación JEL: A12, B53, N50, N70, Q4, Q40.

Abstract: This paper analyses both, the physical concept of Energy and its applications, from the point of view of the Human Action, or Praxeology. In this task, the author applies the analytical toolbox developed by the Austrian School of Economics. In order to make the analysis comprehensible, this paper first develops the concept of Energy from an Ontological and Scientific perspective, and then offers a brief history of its utilisation by the human being to achieve his goals. Once established the point of departure, energy is characterised as the ultimate economic good of superior order, demonstrating the importance of the dynamic efficiency in its study. The close ties with the

* Máster Universitario en Economía de la Escuela Austriaca, Universidad Rey Juan Carlos, Madrid. Especialista en energía. Contacto: email@antonio-espana.es. Dirección: C/ Bahía de Cádiz, 15, 1.ํA. 28042 Madrid. Email: email@antonio-espana.es 
wealth of nations is also demonstrated, as it is the relevance of its role in all stages of the production process.

Key words: Energy and Economics, Energy and Praxeology, History of Energy concept, History of Energy applications, Energy as an economic good, Energy and Wealth.

JEL Classification: A12, B53, N50, N70, Q4, Q40.

\section{I \\ INTRODUCCIÓN}

En una economía tan compleja como la moderna, la energía es un factor de producción clave, dado que es necesaria no sólo para ser consumida en la preparación de las comidas con las que nos alimentamos los hombres, en el mantenimiento del confort de nuestro hogar -iluminación, calefacción-y en hacer funcionar el equipamiento que nos entretiene en los momentos de ocio, sino que es imprescindible para virtualmente cualquier proceso productivo, sea en la fabricación de los propios bienes que consumimos, como en la fabricación de los bienes de capital, extracción de materias primas, etc.

Tan importante es, y tanto se ha acostumbrado el ser humano a utilizarla, que la palabra con la que designamos la energía o cualquiera de sus manifestaciones se ha convertido en un término de uso común por la inmensa mayoría de las personas. Sin embargo, se trata de algo relativamente novedoso, sin lo que hemos vivido la mayor parte de nuestra existencia y cuyo desarrollo no ha despegado sino en los últimos 150-200 años. Se trata, además, de un fenómeno físico que aún no ha sido comprendido en su totalidad y sobre el que los científicos no terminan de ponerse de acuerdo.

No es de extrañar, por tanto, que sea complicado encontrar análisis teóricos rigurosos desde el punto de vista de la economía acerca de lo que la energía, su producción y su uso suponen para la acción humana. La mayor parte, o bien se centran en descripciones tecno-económicas, o bien se ocupan únicamente de 
historia económica. Las más ambiciosas, además, se ven afectadas por los errores típicos de la concepción neoclásica, estática, obsesionada con la búsqueda del óptimo paretiano y, sufriendo de altas dosis de formalismo matemático, se empeñan en crear modelos econométricos cuya principal premisa de partida - el que la información está dada-sabemos por los autores de la escuela austriaca que es falaz.

Por eso resulta necesario realizar un análisis de la energía desde el punto de vista económico que tenga en cuenta los desarrollos teóricos de esta escuela sobre la acción humana, enfocando el tema desde una perspectiva praxeológica que nos permita incluir en su estudio la función empresarial y, con ella, la concepción dinámica de la eficiencia.

Este es el propósito de este trabajo, que es una primera aproximación al tema de la energía desde los postulados de la escuela austriaca que deberá ser ampliada y desarrollada en mayor profundidad en ulteriores trabajos de investigación.

Este primer acercamiento debe comenzar, pues no podría ser de otra forma, con una clarificación de lo que entendemos por el concepto de energía. Para ello, comenzamos por aclarar los orígenes y el significado del término, tanto en el lenguaje común como en el científico propio de la física. A continuación, trazamos un breve bosquejo histórico de la evolución del concepto de energía dentro del campo que le es propio, así como del principio de conservación al que va aparejado - de hecho, la intuición de que hay algo que se conserva es anterior al propio descubrimiento de la magnitud conservada, como veremos.

Introducida la energía y siquiera someramente familiarizados con ella, es necesario hacer un repaso histórico de su aplicación práctica que nos ayude a poner en contexto y resaltar la relevancia del estudio teórico de la energía desde la perspectiva económica que viene a continuación. Esto es así porque lo que nos interesa de la energía no es su definición abstracta, objeto del estudio de la física, sino el uso que el hombre común le da en sus actividades cotidianas.

A continuación, entramos en materia abordando el análisis de la energía como el factor de producción o bien económico de orden superior por excelencia, aplicando en su estudio las leyes 
praxeológicas desarrolladas por la escuela austriaca. Finalmente, concluimos con un resumen de las principales ideas recogidas en el trabajo y llamamos la atención sobre la necesidad de un programa de investigación que, desde la misma perspectiva aplicada en el presente trabajo, analice la intervención actual del estado en todas las actividades económicas relacionadas con la producción y consumo de energía, y proponga un modelo alternativo basado en las reglas estrictas del libre mercado que permitan que la función empresarial alcance su máximo potencial y nos garanticemos un suministro de energía dinámicamente eficiente y que dure siempre.

\section{II}

\section{ENERGÍA: UN CONCEPTO DISCUTIDO Y DISCUTIBLE}

\section{Origen y significado del término «energía»}

Nuestra primera tarea en este trabajo debe ser definir el concepto de energía, algo que, como veremos, no resulta una labor sencilla. Los propios estudiosos de la física tardaron siglos en concretarlo tras no poco debate. De hecho, el concepto de energía desde el punto de vista tecno-económico tal y como lo conocemos hoy, es relativamente moderno con apenas ciento cincuenta años de historia.

El Diccionario de la Real Academia Española define la energía en su primera acepción como «eficacia, poder, virtud para obrar» ${ }^{1}$ y en la segunda acepción, asociada a la ciencia física como la «capacidad para realizar un trabajo».

Etimológicamente, el término «energía» procede del vocablo

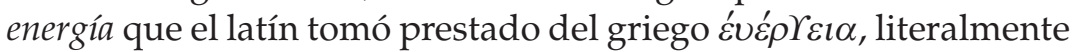
«acción vigorosa», «fuerza en acción» o «acto», que a su vez se derivaba de $\varepsilon \rho \gamma o ́ \zeta$, «obra» ${ }^{2}$ o «trabajo».

\footnotetext{
1 Esta primera acepción resulta especialmente interesante al relacionar directamente el concepto de energía con el de acción humana. No obstante el propósito de este documento es un estudio de la energía económico y no tanto etimológico.

2 V. Corominas (2009) p. 211.
} 
Debemos tomar con cautela esta definición, pues no podemos asumir que los griegos emplearan el término para referirse al mismo concepto o siquiera similar al actual. Así, aunque parece que Aristóteles fue uno de los primeros en darle un uso técnico al atribuirle el significado de «fuerza interior» que movía los seres, y en particular a los seres vivos, en realidad el estagirita utilizó la misma palabra con diferentes sentidos en lugares distintos. Por ejemplo, en su Ética lo usó con el significado de actividad frente a la mera disposición, mientras que en la Retórica lo aplicó para cualificar un estilo vigoroso. De acuerdo con Philip Mirowsky, el filósofo griego también utilizó la palabra «energía» para diferenciar entre actividad y potencialidad, así como para implicar la transformación continúa de la potencia al ser —ad posse ad esse. ${ }^{3}$

La primera documentación de esta palabra en nuestro idioma, según Corominas, se halla en el Tesoro de la lengua castellana o española de Sebastián de Covarrubias, ${ }^{4}$ diccionario de español publicado en 1611 que es considerado como el primer diccionario monolingüe del idioma castellano:

ENERGÍA. La fuerza que encierran en sí algunas palabras preñadas y dichas con cierto espíritu, que nos publican lo que callan. El nombre es Griego, évé $\rho$ rel $\alpha$, vis ex se movens, agitatio, efficacia, seu operatio interior, quae est veluti vi ac dux ad exteriorem corporis actum. ${ }^{5}$

El término «energía» aparece un poco más tarde, en 1632 en la obra La Dorotea ${ }^{6}$ de Lope de Vega, donde llama la atención su

3 V. Mirowsky (1989) p. 13

4 Sebastián Covarrubias (1539-1613), lexicógrafo y escritor español, capellán del rey Felipe II y canónigo de la catedral de Cuenca era además sobrino de Diego de Covarrubias y Leyva (1512-1577) — la madre de Sebastián María Valero de Covarrubias Leyva era prima hermana de don Diego-, uno de los representantes de la Escuela de Salamanca en el Siglo de Oro español. Según Huerta de Soto, Diego de Covarrubias «expuso mejor nadie antes que él la esencia de la teoría del valor, sobre la que gira todo el entramado del análisis económico de la Escuela Austriaca». $V$. Huerta de Soto (2000). Tanto Sebastián como su hermano, abandonaron a su padre biológico por su tío don Diego, quien ejerció su influencia para que ambos ocuparan puestos eclesiásticos de importancia. Ambos Covarrubias mantuvieron, de hecho, una relación cuasi paterno-filial. V. Weiner (2003).

5 V. Covarrubias (1611) p. 473.

${ }^{6} V$. Vega (1632) p. 133 y en especial n. 10. Anotaciones de Edwin S. Morby. 
manera de introducirlo como un neologismo, dando prueba de que en pleno siglo XVII distaba de ser un término de uso común:

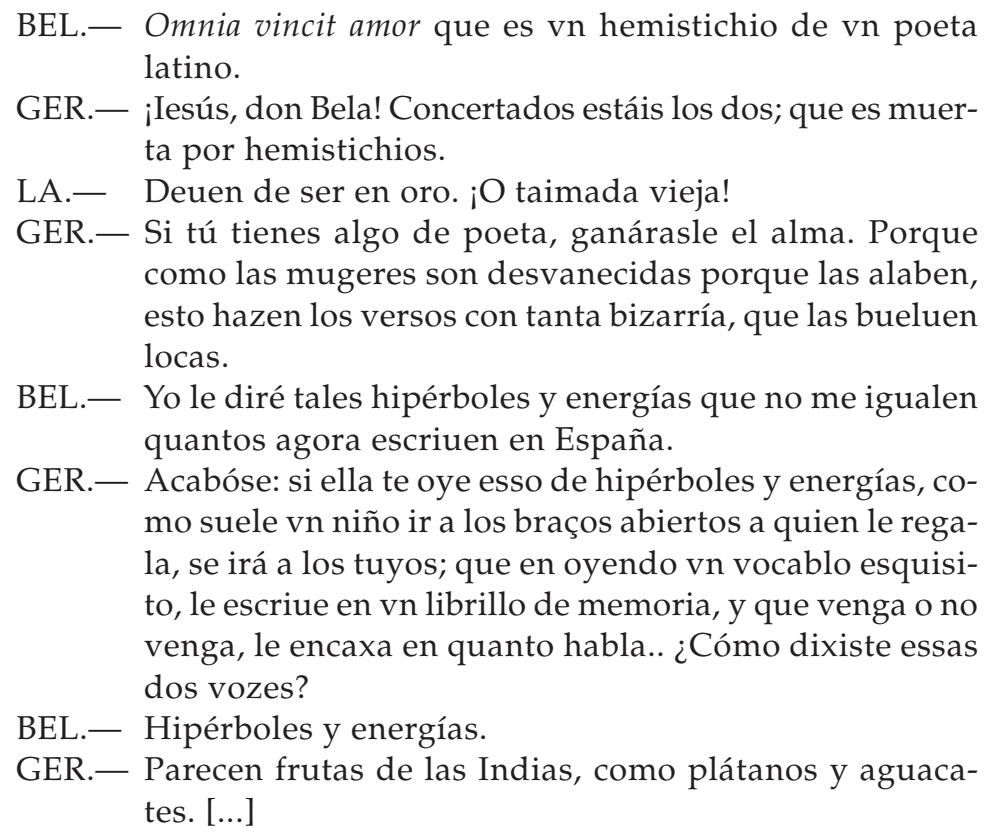

Evidentemente, la definición más precisa del concepto de energía proviene del campo de la física, que la define, como hemos visto arriba, como la capacidad para realizar trabajo. Y si por algo es interesante la energía desde la perspectiva económica, disciplina que nos ocupa, es precisamente por esta capacidad para realizar trabajo que resulte útil para lograr un fin determinado. De hecho, acabamos de ver como esta palabra forma parte de la versión griega del vocablo energía.

Más adelante volveremos sobre la caracterización económica del trabajo al que nos referimos. De momento permaneceremos en el campo de la física, donde el «trabajo» se define como una «fuerza» ejercida a lo largo de una «distancia» determinada. El trabajo, que como magnitud física se expresa en las mismas unidades que la energía, se mide según el Sistema Internacional (SI) en «julios». Un julio es la cantidad de trabajo realizado por 
una fuerza de un newton que actúa a través de la distancia de un metro. El newton que es la unidad del SI para expresar la fuerza se define a su vez como la fuerza necesaria para, en el lapso de un segundo, incrementar la velocidad de una masa de un kilogramo en un metro por segundo. ${ }^{7}$

Otra magnitud muy habitual relacionada con la energía y el trabajo es la potencia, que no es sino la tasa o velocidad a la que el trabajo se realiza, o lo que es lo mismo, el resultado de dividir el trabajo por el tiempo empleado en realizarlo. Así, un watio - la unidad de potencia utilizada en el SI- es la potencia utilizada en realizar un trabajo de un julio en un segundo.

Este concepto físico de energía y su desarrollo científico es muy relevante para la ciencia económica, dado que, como sostiene el profesor Huerta de Soto en su artículo La teoría de la eficiencia dinámica, "tiene una influencia determinante la forma en que el surgimiento y desarrollo de la física mecánica termina afectando a la evolución del pensamiento económico, especialmente a partir del siglo XIX». ${ }^{8}$ Sobre todo en tanto en cuanto la física moderna se construye sobre un concepto discutible y discutido, como veremos en el siguiente apartado. ${ }^{9}$ Philip Miroswki afirma que no es posible entender la economía y las ciencias sociales en el siglo XX sin entender el concepto de «energía» con cierto detalle. Es más, para aprehender correctamente este concepto es necesario familiarizarse antes con la historia de su desarrollo. ${ }^{10} \mathrm{Y}$ esto es precisamente lo que nos proponemos a continuación.

7 De hecho, podemos observar que en el campo de la electricidad, la energía suele expresarse en términos de la potencia entregada multiplicada por el tiempo en el que ésta se utiliza, es decir en watios $x$ hora. Una simple operación aritmética nos indica que un watio.hora es equivalente a 3.600 julios.

8 V. Huerta de Soto (2004) p. 16.

9 A este respecto, afirma Huerta de Soto que se trata de un «concepto abstracto del que todos los físicos hablan y discuten, aunque no se pongan muy de acuerdo sobre lo que exactamente sea la energía, salvo cuando observan sus efectos en forma de fuerza o movimiento. V.op. cit. p. 17.

10 V. Mirowski (1989) p. 11. 


\section{Bosquejo histórico del desarrollo científico del concepto de energía}

Históricamente, el concepto de energía no comienza a cobrar relevancia en la ciencia hasta el siglo XIX, cuando se produjo el desarrollo de la termodinámica y adquirió el carácter unificador, relacionando campos hasta entonces inconexos como la física o la química. De hecho, la historia del concepto de energía sigue un tortuoso camino que parte desde la consideración de ésta como una substancia, pasa luego a convertirse en un mero formalismo matemático en forma de $\operatorname{campo}^{11}$ y termina por considerarse meramente como otra manera de expresar una simetría algebraica.

El concepto de energía está estrechamente vinculado con el principio, esencialmente estático, ${ }^{12}$ de la conservación de la energía en particular y con los principios de conservación en general, sin que pueda explicarse el mismo sin hacer referencia a dichos principios. Nos referimos a lo que a menudo encontramos formulado como el aforismo «la energía ni se crea ni se destruye, sólo se transforma», tal y como es vulgarmente conocido. Esta regla de conservación, según Mirowski en realidad no resulta fácil de definir en términos concisos en su concepción abstracta. ${ }^{13}$ De hecho, afirma este autor que la historia de la ciencia física occidental puede condensarse como la búsqueda previa a 1850 del principio de conservación adecuado para explicar el movimiento, y con posterioridad a ese año, del principio para explicar todos los fenómenos físicos.

Parece estar generalmente aceptado que esta búsqueda de la magnitud que se conserva la inició Descartes en 1644, identificándola erróneamente con la cantidad de movimiento. Posteriormente, en 1695 Leibniz revisó las nociones de fuerza hasta esa fecha

11 En física, un campo representa cualquier magnitud distribuida en el espacio. Inicialmente se introdujo para explicar cómo se ejercen las acciones a distancia entre partículas, como el campo eléctrico o el campo gravitatorio.

12 Estático en el sentido en que se refiere el profesor Huerta de Soto en op. cit. p. 17.

$13 V$. Mirowski (1989) p. 13. Mirowski aporta como «definición provisional» the rule that some particular aspect of a phenomenon remains invariant or unaltered while the greater phenomenon undergoes certain specified transformations. 
y estableció la distinción entre fuerza muerta (vis mortua), que aún no había producido movimiento pero tenía la potencialidad, y fuerza viva (vis viva), que sí se encontraba en acción y que podía ser medida por sus efectos observables. Manteniendo el principio de que la causa debe equivaler a sus efectos, afirmó que esa vis viva se conservaba en el proceso de movimiento.

En 1744 Maupertuis introdujo el principio de minima acción, sugiriendo que el principio de conservación de la vis viva de Leibniz era un caso particular de aquél. Según Maupertuis, la naturaleza es económica en todas sus acciones, implicando esto que siempre se comporta de manera que se minimiza una nueva magnitud, que él mismo introdujo denominándola acción y que definió como masa multiplicada por su velocidad multiplicada por la distancia recorrida. Esta magnitud física no ha llegado a nuestros días pero el principio de mínima acción convulsionó el edificio construido por Leibniz sobre la regla de conservación.

Fue Euler quien en 1752 concilió las concepciones de Leibniz (principio de conservación) con la de Maupertuis (principio de variación), desarrollando el aparato matemático apropiado - cálculo diferencial- y conectando la fuerza con la vis viva según se entienden actualmente. Posteriormente, en 1788, Lagrange perfeccionó las técnicas analíticas y rechazó el principio de mínima acción por considerarlo «metafísico». En cualquier caso, afirma Mirowski que a medida que el aparato matemático crecía en importancia, se oscurecía el significado de las reglas de conservación:

\footnotetext{
Although the appropriate mathematics were in place by the turn of the nineteenth century, it appears that the conceptualization of analysis as extremal principles coupled to conservation principles was not much further (...). This conceptual stasis was rooted in the fact that a science of dynamics, predicated upon the specification of maximization or minimization, was struggling to describe a system undergoing change, yet simultaneously founded upon some unchanging natural phenomenon that would serve as a benchmark; it had yet to suggest a legitimate quantitative conserved unit. ${ }^{14}$
}

\footnotetext{
14 V. Mirowski (1989) p. 23.
} 
Así, a comienzos del siglo XIX no estaba en absoluto claro que la ciencia mecánica fuera capaz de proporcionar un paradigma válido que explicara el mundo físico en general. No fue sino el desarrollo de la ingeniería en las escuelas técnicas de la Francia napoleónica, y sus intentos por formalizar el valor del trabajo, dominar el poder del vapor y estudiar el fenómeno del calor, lo que propició el siguiente gran avance en la comprensión del principio de conservación. Éste se debió al inicio de la termodinámica, cuya fundación se atribuye a Nicolas Léonard Sadi Carnot que, aplicando las ideas de su padre, Lazare Carnot, a la máquina de vapor, reconceptualizó la naturaleza del calor y relacionó ésta con el trabajo mecánico de manera casi definitiva. ${ }^{15}$

Aun así, Carnot no dio todavía con la magnitud correcta que debía conservarse, pues se basó en un supuesto fluido hipotético denominado calórico que entraba en contradicción precisamente con la capacidad de un motor para generar trabajo mecánico.

Es a mediados del siglo XIX, en torno a 1840, en el que los historiadores de la ciencia ubican el descubrimiento simultáneo del principio de conservación de la energía. Fue, por tanto, esa época en la que el término «energía» comenzó a adquirir el sentido por el que hoy lo conocemos. El nuevo concepto se convirtió en el factor unificador de la investigación científica, enlazando entre sí disciplinas que estudiaban fenómenos hasta entonces separados y diferentes: movimiento, luz, calor, electricidad y magnetismo. Y el nuevo concepto fue el que elevó la física a la categoría de disciplina reina de las ciencias, destronando a la astronomía. ${ }^{16}$

Aunque el principio de conservación de la energía pudo tener varios padres, las figuras más relevantes en su descubrimiento simultáneo fueron Julius Robert Mayer, James Joule, Hermann von Helmholtz y Ludwig Colding. Lo que estos cuatro hombres descubrieron se puede descomponer en cuatro ideas según Mirowski:

15 Sadi Carnot descubrió que era la diferencia de temperatura la que provocaba el movimiento, independientemente de la sustancia calentada o enfriada, de manera análoga a un molino de agua movido por la caída del líquido desde una altura superior a una inferior. La demostración de su descubrimiento se basaba en la imposibilidad del movimiento perpetuo, algo que en el primer cuarto del siglo XIX era aún una tesis controvertida y no compartida por todos los científicos.

16 V. Mirowski (1989) p. 35. 
la formación del propio concepto de energía, la afirmación ontológica de que existe una energía esperando a ser descubierta, la formulación matemática de que la energía ni se crea ni se destruye, y el procedimiento para justificar las ideas segunda y tercera.

En cualquier caso, el concepto de energía era aún difuso, dado que permanecía cierta confusión con el concepto de fuerza y aún se contraponían las concepciones de la energía como sustancia y como acción. Es más, por «energía» se hacía referencia a un conjunto diverso de conceptos que había de ser consolidado y concretado en una única «cosa». Esto no se consiguió hasta los años 50 del siglo XIX, siendo el mérito de su correcta definición atribuido a William John Macquorn Rankine:

the term energy is used to comprenhed every affection of substances which constitutes or is commensurable with a power of producing change in opposition to resistance, and includes ordinary motion and mechanical power, chemical action, heat, light, electricity, magnetism and all other powers, known or unknown, which are convertible or commensurable with these. All conceivable forms of energy may be distinguished into two kinds; actual or sensible, and potential or latent. Actual energy is a measurable, transferable and transformable affection of substance to change its state in one or more respects; by the occurrence of which changes, actual energy disappears, and its replaced by potential energy (...) The law of the conservation of energy is already known, viz. that the sum of the actual and potential energies in the universe is unchangeable. ${ }^{17}$

Pero esta visión de que la energía se puede transformar y convertir de una a otra forma conservándose la cantidad total existente, parece contradecir el hecho de que nos encontramos en un mundo que es sustancialmente irreversible: no existen los motores $100 \%$ eficientes - en el sentido que son capaces de aprovechar toda la energía contenida en el combustible-, no se puede restituir un tronco quemado a partir de sus cenizas, no se puede mantener un objeto en movimiento perpetuo a causa del rozamiento, p. 96.

17 Citado por Mirowski en op. cit. p. 53, que a su vez toma la cita de Lindasy (1976) 
ni tampoco podemos hacer retroceder nuestro envejecimiento y volvernos más jóvenes. El problema, por lo tanto, se trasladó al intento de reconciliar esta nueva noción de energía con las teorías existentes acerca del calor. Fue William Thomson quien descubrió que esta reconciliación requería de otro principio aparte del de conservación de la energía y que además no contradijera el principio descubierto por Carnot: el de que el calor sólo puede viajar de un objeto caliente a otro más frío y no a la inversa. ${ }^{18}$

Ambos principios fueron reformulados en 1865 por Rudolph Clausius, que introdujo el concepto de entropía como medida o grado de desorden de la materia y estableció lo que hoy en día se conocen como la primera y segunda leyes de la termodinámica respectivamente: (1) que la energía es constante en el universo y (2) que la entropía del universo está en permanente crecimiento hasta que se alcance su nivel máximo. En términos prácticos, lo que el segundo principio nos viene a decir es que existen dos tipos de intercambio de energía: uno en el que la nueva forma de energía es capaz de realizar un trabajo - el del ciclo de Carnot mencionado arriba- y otro en el que la energía se «disipa» 0 «desperdicia», convirtiéndose en una clase de energía que no puede utilizarse para realizar ningún tipo de trabajo.

Esto último resulta ser una contradicción clara con la definición de energía dada al comienzo de este capítulo — capacidad para realizar un trabajo. Efectivamente esta aparente contradicción no pasó desapercibida a los científicos de la época, que se embarcaron en la tarea de resolverla. Fue así como se introdujeron en el estudio de la energía la teoría de la probabilidad - que daría lugar a la mecánica cuántica-y formalismos matemáticos complejos como la teoría de campos o la teoría de grupos - que a la postre culminaron con la teoría de la relatividad.

No concluye aquí la historia del desarrollo científico del concepto de energía aunque para nuestro propósito es suficiente detener en este punto su exposición, ya que nos basta con la termodinámica clásica para continuar con nuestro análisis teórico

18 De estos dos principios se deduce la idea de que el Universo se encamina a un estado final de equilibrio térmico en el que no existan ya diferenciales de temperatura del que extraer trabajo mecánico de ningún tipo. Sería la muerte del Universo. 
económico. No entramos a relatar, siquiera someramente, los avances ulteriores dado su elevado nivel de abstracción y formalismo matemático, pues complicarían la exposición sin aportar elementos que faciliten la comprensión del resto del texto. Además, su relación con la visión del mundo macroscópico y fenomenológico, que es el nos interesa desde el punto de vista económico - quizás con la excepción de los estudios que han permitido el desarrollo de la energía nuclear - es todavía escasa o nula, y más que haberse consolidado en una teoría comprensiva de la energía y su conservación, el desarrollo reciente de la física de la energía ha constituido una diáspora en direcciones opuestas. En palabras de Philip Mirowski:

the twentieth century has transmuted it beyond all recognition and coherence. First in the theory of relativity, then in quantum mechanics, and finally in modern cosmology and particle physics, energy was turned on its head. Each new innovation served to qualify and transform the conservation law further and further, until no one is willing to state definitely and specifically what has been preserved. ${ }^{19}$

O como más adelante concluye el mismo autor, condensando en una frase la historia del concepto:

Energy, it seems, has finally become a purely instrumental and mathematical entity, transformed along its history from a substance to an entity to a relation to, finally, a gestalt. ${ }^{20}$

III

\section{BREVE HISTORIA DE LA APLICACIÓN PRÁCTICA DE LA ENERGÍA}

Hemos repasado en el capítulo anterior el concepto de energía, el origen del término y la historia del desarrollo de su concepción científica dentro del campo de la física. Sin embargo, decíamos

\footnotetext{
19 V. Mirowski (1989) pp. 76-77.

20 Ibídem p. 93.
} 
que nos interesa la energía, no como concepto abstracto y objeto del estudio de personas con bata blanca y confinado a los laboratorios de física, los aceleradores de partículas y los complejos artificios matemáticos, sino por el uso que el hombre común le da en sus actividades cotidianas. Es decir, nuestro interés en la energía es económico, o más específicamente, praxeológico.

Pero antes de adentrarnos en el terreno de la teoría económica pura, merece la pena realizar un repaso histórico de la aplicación práctica de la energía que nos ayude a poner en contexto y resaltar la relevancia de su estudio teórico desde la perspectiva económica.

El primer hito relevante en la historia del uso de la energía por parte del hombre fue el descubrimiento del fuego, o más precisamente, la invención de una manera de controlar el fuego. Suele datarse este hecho en algún momento del paleolítico hace entre ochocientos y cuatrocientos mil años. ${ }^{21}$ Antes de contar con el fuego, el hombre sólo contaba con la fuerza de sus propios músculos como única fuente de poder para realizar cualquier trabajo.

Para el siguiente hito importante tenemos que avanzar otros cientos de miles de años hasta que el hombre comenzó a domesticar animales hace unos doce mil años. ${ }^{22}$ De este modo, aprovechando la potencia muscular de los animales más grandes y más fuertes, el hombre logró mayores cotas de productividad: podía labrar la tierra más rápido que con un simple arado manual y podía moverse más rápido y transportar más bienes que a cuestas o tirando de un carro. El incremento de la productividad y

21 Parece que hay cierto desacuerdo en establecer cuál es la evidencia más temprana del uso del fuego por el humano o alguno de sus predecesores, siendo el caso más antiguo el del emplazamiento de Gesher Benot Ya'aqov en Israel, datado hace setecientos noventa mil años y en el que se encontraron semillas y fragmentos de madera quemados. El siguiente caso en competencia es bastante más reciente, con cerca de cuatrocientos mil años de antigüedad. Se trata del yacimiento de Zhoukoudian en China, donde junto con utensilios de cuarcita se encontraron restos de huesos de animales que hacen pensar que fueron quemados de manera controlada.

${ }^{22}$ Las primeras evidencias aparecen de nuevo en China y Asia, donde parece que el hombre había domesticado perros hace doce mil años. Las ovejas, cabras y cerdos se convirtieron en ganado hacia el año 8000 AC, el bovino hacia el 6000 AC y los caballos, mulas y bueyes en torno al 4000 AC. $V$. Diamond (1997) p. 167, citado por Bradley y Fulmer (2004) p. 11. 
la acumulación de capital que este avance permitió —a su vez potenciado por el surgimiento de instituciones sociales clave como el dinero o el lenguaje- facilitó, sin duda, el surgimiento de las primeras grandes civilizaciones.

La primera industria que usó intensivamente la energía para funcionar fue la metalurgia, comenzando por el cobre hace unos cinco mil años. Esto fue así porque con una temperatura de fusión relativamente baja (en torno a 1.100 grados centígrados), resultaba más sencillo de fundir y moldear con hornos rudimentarios que otros minerales más abundantes y accesibles. El cobre sería posteriormente aleado con estaño para formar el bronce. ${ }^{23}$

Con posterioridad, y a medida que avanzó la tecnología para el mejor aprovechamiento de la energía, comenzó a trabajarse en los talleres metalúrgicos el hierro, mineral más abundante que el cobre pero más difícil de manejar por su punto de fusión más elevado (unos 1.500 grados). No obstante, hacia la Edad Media, la metalurgia del hierro ya se había extendido por todo el occidente de Europa. La generalización de la demanda de productos fabricados con hierro y, por lo tanto, la extensión de su producción, conjuntamente con el relativamente bajo poder calórico de la madera empleada en las fraguas, originó en Inglaterra una tala masiva de árboles y la consiguiente escasez de madera. Esto propició que se comenzara a utilizar el carbón como combustible y que se desarrollara en aquel país la minería del carbón a partir del siglo XVII. ${ }^{24}$

${ }^{23}$ Podría decirse que el uso industrial del cobre gracias a la metalurgia fue de crucial importancia para el desarrollo de la sociedad, no únicamente por los bienes de capital que podían producirse, sino debido también a que, de alguna manera, fue parcialmente culpable de la extensión del comercio y con él, de las técnicas de producción desde Oriente Próximo a los pueblos bárbaros de Europa septentrional. Asimismo, el comercio de este metal podría también explicar, siquiera parcialmente, los procesos de colonización fenicios y griegos de la Europa meridional.

${ }^{24}$ Julian Simon en su The Ultimate Resource 2 recoge este hecho: «the shortage of wood for use as charcoal in the casting of iron became so acute -it was affecting the building of naval ships - that in 1588 Parliament passed a law against cutting trees for coke in iron making, and then banned the building of new foundries in 1580 . Though the use of coal in place of charcoal had been known, there were technical difficulties -impurities that affected the quality of the iron. This time, the wood shortage exerted pressure that led to the development of coal as well as blowing machines to be used in smelting, a keystone in the upcoming Industrial Revolution». V. Simon (1996) p. 169. 
Así, podemos observar cómo durante la mayor parte de la historia de la humanidad, el hombre ha satisfecho sus necesidades energéticas mediante fuentes de energía vegetales ${ }^{25}$ : bien sea quemando madera para calentar e iluminar sus estancias, forjar el hierro o cocer los utensilios de barro, bien sea para alimentar caballos, bueyes y otros animales empleados en el transporte o la labor de la tierra.

Esta situación fue así incluso durante una porción muy significativa de nuestra era, dado que, con la excepción de las invenciones del molino de viento y de agua ${ }^{26}$ y de la navegación a vela, el desarrollo tecnológico del uso mecánico de la energía no despegó hasta que se inventó la máquina de vapor en el siglo XVII. Como sostienen Bradley y Fullmer:

with possible exceptions of the sail, the windmill, the waterwheel, and gunpowder, the technology used by the average person did not change much for thousands of years. Romans living at the time of Christ would have easily understood the science of the $16^{\text {th }}$ Century. ${ }^{27}$

Como consecuencia de la generalización del uso de la máquina de vapor que trajo consigo - o más bien fue protagonista de- la Revolución Industrial, durante el siglo XIX se produjo la extensión del carbón como fuente de energía, provocando un incremento exponencial de la demanda del carbón ${ }^{28}$ que a su vez

25 Hoy conocidas pomposamente como biomasa.

26 Los primeros molinos de agua del tipo horizontal, de hecho parece que existían en la antigua Mesopotamia (6000 - 5000 AC), mientras que los de tipo noria en la que el agua cae verticalmente se inventaron en Oriente Próximo en el siglo IV de nuestra era, desde donde fueron introducidos en Europa por los árabes a través de España. Parece, asimismo, que los molinos de viento fueron inventados en China e introducidos en Europa occidental en el siglo XII. V. Barquín (2004) n. 6 p. 20.

27 V. Bradley y Fullmer (2004) p.11.

28 Tanta importancia cobró la utilización del carbón como combustible, que un economista de la talla de William Stanley Jevons, con la publicación en 1865 de su Coal Question, podría decirse que inauguró todo un género literario: el del panfleto alarmista que predice el agotamiento de los recursos energéticos del mundo, $V$. Jevons (1866). El precedente de las predicciones agoreras del agotamiento de recursos vitales para la vida, no obstante, lo sentó Thomas Malthus en 1798 cuando adelantó que la tierra se quedaría sin alimentos para satisfacer toda la población. 
impulsó y realimentó la evolución de las técnicas de explotación minera y de la ingeniería. Así, a finales del siglo XIX se inventó la turbina de vapor moderna, en cuyo principio se basa gran parte de la producción de electricidad actual. De hecho, durante el siglo XX el principal uso energético del carbón fue precisamente la producción de energía eléctrica.

El perfeccionamiento de la turbina de vapor a su vez y junto con los avances de la tecnología eléctrica y de sus aplicaciones, impulsó el desarrollo tecnológico necesario para la incorporación de otros combustibles durante el siglo XX, como el gas o el petróleo que han ido sustituyendo gradualmente al carbón como fuente de energía. Igualmente, este mismo desarrollo de la tecnología eléctrica ha permitido a lo largo de los últimos cien años aprovechar y utilizar la energía procedente de centrales hidráulicas, nucleares, eólicas o solares.

Todo ello sin olvidar al petróleo, protagonista indiscutible en la historia de la energía del siglo XX, empleado en la generación de electricidad pero, sobre todo, en el transporte gracias a la invención a finales del siglo XIX del motor de explosión.

\section{IV \\ ENERGÍA: UNA VISIÓN PRAXEOLÓGICA}

En su obra The Ultimate Resource 2, Julian Simon sostiene que

Energy is the master resource, because energy enables us to convert one material into another. ${ }^{29}$

$Y$ en efecto, dado que se requiere la utilización de energía para la producción de cualquier bien, sea de capital o de consumo y dado que ésta es un factor de producción completamente inespecífico - pues, en esencia, es la misma energía la que se emplea en iluminar las instalaciones de una fábrica, que la que se usa para hacer funcionar su maquinaria, que la que utilizan

\footnotetext{
29 V. Simon (1996) p. 162.
} 
los ordenadores del departamento de ingeniería y las aspiradoras del servicio de limpieza de las oficinas-, podemos afirmar según la teoría económica de los bienes que la energía es el factor de orden superior por excelencia.

Esto es lo que demostraremos en las siguientes líneas, estableciendo las relaciones entre la caracterización de la energía desde el punto de vista de la ciencia física y, concretamente, de la rama de la termodinámica, y la teoría económica o praxeología según ha sido enunciada por los teóricos de la escuela austriaca.

\section{1. ¿Es la energía un bien económico?}

Carl Menger, en el primer capítulo de sus Principios de economía política explica su teoría general del bien. Haciendo aplicación del principio de causalidad, define las cosas útiles como aquellas "cosas que tienen la virtud de poder entrar en relación causal con la satisfacción de las necesidades humanas» y, «en la medida en que reconocemos esta conexión causal y al mismo tiempo tenemos el poder de emplear las cosas de que estamos hablando en la satisfacción de nuestras necesidades, las llamamos bienes». En este sentido, enumera cuatro condiciones para que una cosa pueda considerarse un bien: (1) que exista una necesidad humana, (2) que sus cualidades o características permitan establecer una conexión causal con la satisfacción de dicha necesidad, (3) que el hombre conozca esa relación causal, y (4) que se pueda disponer de la cosa en cuestión para utilizarla en la satisfacción de la necesidad. ${ }^{30}$

En términos de la acción humana o praxeológicos, esto equivale a decir que el fin del hombre actor es la satisfacción de una necesidad o, lo que es lo mismo, aliviar una situación de malestar, y el bien es el medio, que es todo aquello que el actor cree subjetivamente que es adecuado para lograrlo. ${ }^{31}$

Además, Menger establece la distinción entre «bienes económicos» $\mathrm{y}$ «bienes no económicos», siendo los primeros aquellos

${ }^{30} \mathrm{~V}$. Menger (1871) pp. 103-104. Las cursivas en las citas corresponden al texto original.

${ }^{31} V$. Mises (1949). Dice Mises que «los medios no aparecen como tales en el universo; en nuestro mundo, tan sólo existen cosas; cosas que, sin embargo, se convierten 
para los cuales su necesidad es superior a la cantidad disponible, «en contraposición a aquellos otros de los que los hombres no tienen ninguna necesidad para su actividad económica» ${ }^{32}$-que serían los bienes no económicos o bienes libres. Menger denomina, pues, economía al conjunto de actividades orientadas a satisfacer las necesidades - lograr los fines - utilizando «las cantidades de bienes de consumo directo, y sobre todo las cantidades de medios de producción de que disponen, de una manera objetiva y racional, para satisfacer sus necesidades del mejor modo posible.» ${ }^{33}$

Por lo tanto, el carácter económico o no de un bien, en cuanto a medio para satisfacer un fin, se establece en la medida que éste sea escaso. Es decir, los medios «por definición han de ser escasos, puesto que si no fueran escasos ni siquiera serían tenidos en cuenta a la hora de actuar. Es decir, allí donde no hay escasez no hay acción humana.» ${ }^{34}$

Realizadas estas acotaciones relativas a la teoría económica cabría preguntarse cómo son de aplicables al concepto de energía que hemos introducido en los capítulos anteriores y si podemos hablar de la energía como un bien económico.

Por un lado, es evidente que la energía, aun en su forma más abstracta, bajo la definición tradicional que vimos al principio como «capacidad para realizar un trabajo» es un medio en el sentido praxeológico siempre y cuando, claro está, haya un ser humano de por medio actuando intencionadamente para lograr un fin concreto: aquel que corresponde al trabajo - en sentido físico-que la energía permite realizar. ${ }^{35}$

en medios cuando, mediante la razón, advierte el hombre la ideonidad de las mismas para atender humanas apetencias, utilizándolas al objeto». V. op. cit. p. 111.

$32 \mathrm{~V}$. Menger (1871) p. 150

33 Ibídem p. 149

$34 V$. Huerta de Soto (2005) p. 44. Cita el profesor Huerta de Soto en la nota 6 a La acción humana de Mises, cuyo pasaje completo reproducimos a continuación: «Los medios resultan siempre escasos, es decir, insuficientes para alcanzar todos los objetivos a los que el hombre aspira. De no ser así, la acción humana se desentendería de ellos. El actuar, si el hombre no se viera inexorablemente cercado por la escasez, carecería de objeto». $V$. Mises op. cit. p. 112.

35 Es decir, la energía asociada al trabajo realizado por la caída del agua en un salto en una remota isla desierta del Pacífico no podemos considerarla un medio de 
Dado por aceptado el hecho de que la energía es un medio que sirve a multitud de fines humanos - calentarse, cocinar, desplazarse, transportar bienes, etc.--, es el turno de demostrar que éste es un factor escaso en el mundo, de forma que podamos considerarla como un bien económico o, lo que es lo mismo, certificar su naturaleza de medio en sentido praxeológico.

Para tal demostración, recurriremos a la ciencia física, y en concreto a la 1. ${ }^{\underline{a}}$ Ley de la Termodinámica, también conocida como el principio de conservación de la energía, y que ha sido comentada en apartados anteriores. Esta ley establece que «la cantidad total de energía en cualquier sistema aislado permanece invariable con el tiempo, aunque dicha energía puede transformarse en otra forma de energía». Llevada a sus últimas consecuencias, este principio nos conduce a afirmar que existe una cantidad limitada - aunque desconocida- de energía en el universo.

En su versión más conocida, este principio viene a decir que «la energía ni se crea ni se destruye, sólo se transforma». Expresado así, parecería que no ha lugar conferirle la cualidad de bien económico en términos praxeológicos, dado que si bien es cierto que hay una cantidad finita en el universo y no hay posibilidad de incrementar el stock de la misma, no deja de afirmar el mismo principio que ésta tampoco se consume ${ }^{36}$.

Para poder hablar de escasez en términos absolutos, debemos recurrir a la $2 .{ }^{\underline{a}}$ Ley de la Termodinámica, también comentada con anterioridad y que determina que «la cantidad de entropía de cualquier sistema aislado termodinámicamente tiende a

ninguna manera. Lo sería si en aquella isla recalara un naufrago al modo de un Robinson Crusoe y, construyendo algún mecanismo rudimentario, aprovechara esa energía para un trabajo más concreto: por ejemplo afilar un trozo de hierro a modo de cuchillo para recolectar moras, o moler semillas silvestres para fabricar alguna especie de harina. El ejemplo de Robinson Crusoe es utilizado por el profesor Huerta de Soto en su Dinero, crédito bancario y ciclos económicos para explicar la teoría del capital, y lo toma a su vez de Eugene von Böhm-Bawerk. V. Huerta de Soto (2009) pp. 218-227.

36 En este aspecto, sería como las obras maestras de la literatura o la música si imagináramos por un momento que ya nunca más naciera un genio capaz de producir nuevas creaciones: el stock de obras maestras sería limitado, pero el hecho de disfrutar del Quijote, por ejemplo, no implicaría que esta novela desapareciera de la faz de la tierra. 
incrementarse con el tiempo». En otros términos, lo que este principio viene a afirmar es que los procesos térmicos fluyen en una única dirección y son irreversibles, en el sentido que la transferencia de energía en forma de calor de un cuerpo a otro siempre se realizará del cuerpo más caliente al más frío, estando prohibido el camino inverso. ${ }^{37} \mathrm{O}$ lo que es lo mismo, la segunda ley afirma la imposibilidad teórica de la máquina de movimiento perpetuo ${ }^{38}$.

La consecuencia de esta aserción es que una vez alcanzado el equilibrio térmico, se detendría la transferencia de energía y, por lo tanto, ningún trabajo útil podría ya extraerse. La cantidad de energía seguiría siendo la misma de acuerdo con el primer principio de la termodinámica, sin embargo, habría una parte que se ha disipado en forma de calor y no sería ya reutilizable para producir ningún trabajo mecánico o de otro tipo. A efectos prácticos y económicos, dicha energía se habría «consumido» para el uso por los hombres.

Así pues, considerando que la energía, a diferencia de los beneficios empresariales puros, no se puede crear ex nihilo y que además con su utilización para lograr un fin determinado, se consume a efectos prácticos, es decir, no es posible emplear esa misma energía en lograr otros fines, podemos afirmar el carácter escaso y, por tanto, de bien económico de la misma.

37 Una analogía que gráficamente facilita la comprensión de esta ley podría ser la de dos vasos comunicantes, en los que el líquido siempre fluye del recipiente que se encuentra más lleno hacia el que se encuentra más vacío. La transferencia de líquido se realiza siempre en esa dirección hasta que ambos vasos alcanzan el mismo nivel —se alcanza el equilibrio. Espontáneamente es imposible que sucediera al contrario, habríamos de emplear energía procedente del exterior del sistema para invertir el flujo del líquido - acción que se suele realizar y que es la base de los sistemas hidráulicos, al igual que los sistemas de refrigeración extraen calor del aire utilizando el aporte de la energía eléctrica.

38 Aún existe una tercera ley que declara que es imposible alcanzar una temperatura igual al cero absoluto mediante un número finito de procesos físicos. Discutido y discutible su carácter de ley científica, tiene también escasa repercusión en nuestro análisis praxeológico. 


\section{La energía como bien económico de orden superior}

Retomando la teoría general del bien enunciada por Menger, debemos hacer referencia ahora a su explicación sobre la conexión causal de los bienes y la utilización de esta relación como criterio para clasificar éstos en bienes de primer orden, segundo orden, etc. Menger define como bienes de primer orden todos aquellos para los que existe una conexión causal directa con la satisfacción de nuestras necesidades. Es decir, entrarían en esta categoría de bienes los alimentos y bebidas, la ropa para vestirse, los objetos de adorno, etc. ${ }^{39}$

Los bienes de segundo orden serían aquellos que «sin tener la cualidad de proporcionar la satisfacción inmediata de las necesidades humanas, sirven para la producción de bienes del primer orden y para insertarse, por tanto, en una relación causal mediata respecto de la satisfacción de tales necesidades». ${ }^{40}$

Dado que únicamente con éstos bienes de segundo orden no es suficiente en una compleja economía moderna, debemos hablar de bienes de tercer orden, cuarto orden y así sucesivamente para referirnos a aquellos que se encuentran progresivamente más alejados de la satisfacción directa de las necesidades. Así, Menger pone el ejemplo del pan como bien de primer orden, que sirve al fin inmediato de aliviar el hambre, y la harina o el trabajo del mozo de tahona como bienes de segundo orden, que no satisfacen directamente el apetito pero son necesarios para producir el pan que sí lo hace. Siguiendo por la cadena causal, tendríamos el trigo, el molino o el trabajo del molinero como bienes de tercer orden, el campo de cereales, los aperos de labranza o el trabajo del agricultor como bienes de cuarto orden y así sucesivamente.

No obstante, es necesario recordar la advertencia que hace el propio Menger con respecto a esta clasificación en la que nos previene acerca del error de considerarla una cualidad innata de las cosas. En efecto, ya hemos visto que las cosas no son intrínsecamente bienes, sino que es necesario el concurso de la mente del

\footnotetext{
$39 V$. Menger (1871) p. 108-111.

40 Ibídem p. 109.
} 
sujeto actor, que subjetivamente considera una determinada cosa útil para lograr un fin, para considerarla un bien. Pues bien, la misma intervención de la mente es necesaria para considerar que un determinado bien lo sea de primer orden, de segundo orden o de algún orden superior. ${ }^{41}$

¿Cómo cabría clasificar pues a la energía según lo expuesto anteriormente? Parece claro que debemos considerar a la energía como un bien económico de orden superior, dado que prácticamente siempre es necesario su concurso para la producción de cualquier tipo de bien. El lugar que ocupa en la cadena causal, no obstante, depende del caso y suele ser múltiple. ${ }^{42} \mathrm{Si}$ lo ilustramos con el mismo ejemplo de Menger, podemos ver fácilmente cómo la energía es necesaria para calentar el horno en el que se cuece el pan, pero también para iluminar la panadería donde lo compramos. Si vamos dando pasos previos, veremos cómo también necesitamos energía para mover la rueda del molino con la que hacemos la harina, y también para transportar los sacos de trigo desde el silo de grano hasta el molino, para mover el arado con el que se labra la tierra, para trabajar el hierro con el que se fabrican los aperos de labranza, para calentar la forja, para transportar el combustible de ésta...

En términos físicos, esta caracterización de la energía como bien de orden superior que es necesario para virtualmente cualquier proceso de producción, ${ }^{43}$ se justifica en uno de los corolarios de

41 Ibídem p. 110. Si seguimos utilizando el ejemplo del pan, podemos ver fácilmente como éste puede considerarse como bien de primer orden si lo contemplamos como un alimento para el consumo final, pero también puede ser un bien de segundo orden si nuestro propósito es emplearlo en alguna receta para cocinar un alimento más elaborado, como por ejemplo un gazpacho. Puede consultarse una exposición más resumida que la de Menger acerca de esta clasificación en Mises (1949) p. 113 o en Huerta de Soto (2009) p. 215.

42 De hecho, si no consideramos la energía en sentido abstracto sino en el alguna de sus formas concretas, por ejemplo, el calor, incluso podríamos considerar la energía como un bien de primer orden, dado que iría encaminada a satisfacer una necesidad humana inmediata: la de calentarse.

${ }^{43}$ Hasta en el ejemplo de Robinson Crusoe mencionado en la nota 34 más arriba, se necesitaría una cierta cantidad de energía para recoger las moras necesarias para el consumo diario más básico. En este caso esa cantidad de energía requerida sería la que aportarían las moras recogidas y consumidas el día anterior. 
la segunda ley de la termodinámica, según el cual cualquier alteración del equilibrio físico (como siempre es la producción de un bien) requerirá necesariamente de la alimentación de energía del exterior.

La energía, no obstante, se encuentra en el universo de diferentes formas y con distinta naturaleza: calor, luz, gravitación, nuclear, etc. Pero desde el punto de vista de la economía y relevante para nuestra discusión, el aspecto que más nos interesa es la transformación de la energía de un tipo a otro y su conversión en trabajo o calor útiles para la consecución de los fines de la acción humana. ${ }^{44}$

En este sentido, los sistemas termodinámicos sólo pueden interaccionar (transferir energía) de tres formas (conocidas) diferentes: interacción material o química, interacción en forma de trabajo mecánico o eléctrico e interacción térmica o transferencia de calor. ${ }^{45}$

El primer tipo de interacción, simplificando mucho, es el que se realiza en las reacciones químicas. En esta categoría se incluyen, por ejemplo, los procesos que tienen que ver con el comportamiento fundamental de la materia viva, como por ejemplo el mecanismo de la fotosíntesis, por el que las plantas convierten el agua que toman de las raíces y el ambiente y el dióxido de carbono que cogen del aire, en hidratos de carbono con el que se generan nuevos tejidos orgánicos — hojas, frutos, raíces, saviay oxígeno que devuelven al aire. Para que esta transformación química pueda darse, se necesita un aporte de energía del exterior que les llega en forma de luz solar.

En el terreno de la acción humana, el hombre aprovecha este mecanismo de manera extensiva en todos los procesos de trans-

${ }^{44}$ De la misma forma que la mayoría de los problemas que resuelve la ingeniería son, en esencia, la transformación de energía almacenada a flujos de trabajo y calor que sean útiles para el desarrollo de las actividades humanas, o que, a su vez, puedan transformarse en energía almacenada para su posterior transformación en trabajo o calor útiles.

45 Aunque, como nos indica el segundo principio de la termodinámica, no existen transferencias puras de una a otra forma de energía: siempre habrá una parte de la misma que se transforma — se disipa- en forma de calor que no se puede transformar en ningún otro tipo de energía aprovechable. 
formación de las industrias química y alimentaria. En última instancia, se trata de forzar la realización de reacciones químicas de manera controlada, para las cuales se requiere energía, sea en forma de calor ${ }^{46}$ - hornos- o en forma mecánica - mezcladoras, batidoras o centrifugadoras.

La interacción en forma de trabajo mecánico es la que se produce al impactar dos objetos en movimiento o un objeto en movimiento con uno fijo. Es por ejemplo el tipo de interacción que se produce en un molino de agua, en el que el agua en movimiento -impulsada por la conversión de energía potencial gravitatoria en cinética- choca con las aspas de la noria y la hace girar. Este giro, no es sino un trabajo mecánico que el ser humano aprovecha para moler el grano de trigo y convertirlo en harina.

El trabajo eléctrico es el que se produce cuando se desplaza una corriente de electrones a través de un material conductor de la electricidad. Este tipo de trabajo, por sí sólo no es útil para el hombre directamente pero supone un medio potentísimo para transportar energía de un punto a otro de manera muy rápida - casi instantánea- y, gracias a los avances tecnológicos, el ser humano la ha convertido en una forma de energía muy versátil, pues somos capaces de convertirla en calor - radiadores, hornos eléctricos-, luz — bombillas de todo tipo-, trabajo mecánico - motores electromagnéticos- y reacciones químicas electrólisis. Todas estas formas de energía que se obtienen de la electricidad, pueden a su vez utilizarse directamente para satisfacer fines o bien ser un medio para facilitar otro tipo de interacción termodinámica.

Finalmente, la transferencia de calor es la más habitual y la que dio origen al estudio de la termodinámica ${ }^{47}$. El calor puede transferirse de un objeto - o un gas- a otro mediante el contacto físico (conducción térmica), radiación (emisión electromagnética)

46 El acto de freír un huevo, en el extremo, es provocar un proceso químico denominado desnaturalización, por el que las moléculas que forman la clara del huevo, que en su estado natural — crudo- se configuran de forma viscosa e incolora, por efecto de la temperatura se descomponen para formar otro compuesto diferente, cuyas características principales son que es sólido y de color blanco.

47 En esencia, el término «termodinámica» es un compuesto de dos palabras que viene a significar movimiento del calor. 
o por convección (utilizando un fluido como vehículo). Por ser el tipo de energía empleado de más antiguo, no parece necesario insistir en su utilidad para la consecución de fines humanos, sea de forma directa — calefacción - o indirecta - para ser convertida en otros tipos de energía.

En definitiva, lo que pretendemos justificar con la discusión anterior sobre los diferentes tipos de energía así como de los mecanismos de interacción es que toda energía se puede transformar en otra de otro tipo ${ }^{48}$ y por lo tanto, podríamos asegurar que como bien económico, se trata del más inespecífico de todos los existentes.

Unido este hecho a la afirmación anterior sobre su presencia en cualquier proceso de producción, podemos asegurar que la energía es el bien económico de orden superior por excelencia.

\section{Eficiencia dinámica frente a la eficiencia estática en la energía}

En las secciones anteriores hemos podido comprobar cómo la energía es la magnitud física por antonomasia y el bien económico de orden superior por excelencia. No obstante lo cual, por el hecho de que estemos combinando en nuestro análisis el enfoque estrictamente físico con el económico, no implica que debamos darle un tratamiento mecanicista al mismo.

Porque efectivamente ese es el riesgo que corremos si tenemos en cuenta que,

la economía neoclásica se desarrolla como una copia de la física mecánica del siglo XIX, con unidad de técnica formal, sustituyendo el concepto de energía por el de utilidad y aplicando los mismos principios de conservación, maximización del resultado y minimización del despilfarro. ${ }^{49}$

48 De forma más o menos eficiente y siempre cumpliendo las leyes de la termodinámica, o lo que es lo mismo, produciéndose una «pérdida» de la misma en cada transformación.

${ }^{49} \mathrm{~V}$. Huerta de Soto (2004) p. 18. En este párrafo, el profesor Huerta de Soto hace referencia a los trabajos de Hans Mayer y Philip Mirowski. Éste último aparece citado in extenso en el presente trabajo. 
En efecto, mientras la primera de las leyes de la termodinámica tiene un carácter esencialmente estático - la energía ni se crea ni se destruye-, la segunda determina que la ingeniería mecánica se construya prácticamente sólo en torno al concepto de eficiencia energética, entendida como la minimización del despilfarro de energía. ${ }^{50}$ Se trataría pues de una concepción estática de la energía, al considerar que toda la energía en el universo está dada.

A este respecto, para el profesor Huerta de Soto la influencia de la física mecánica en la ciencia económica es muy negativa dado que

hace que desaparezca la dimensión creadora y especulativa que, desde sus orígenes, tenía el concepto de eficiencia económica. ${ }^{51}$

En nuestra discusión sobre la energía, hemos visto cómo, en efecto, la energía existente en el universo es constante y que, siempre que la transformemos de un tipo a otro hay una pérdida en forma de energía no aprovechable. Sin embargo, no nos interesa la totalidad de la energía que hay en el universo en forma bruta sino aquella que se puede convertir en un trabajo que resulte útil a la consecución de los fines de las personas. ${ }^{52}$

A nuestros efectos, debemos considerar la energía exclusivamente en la medida en que ésta es combinada por el hombre con la tecnología existente en cada momento - es decir, con el conocimiento humano. Así, aunque a efectos prácticos de acción humana, la energía bruta total contenida en el cosmos puede considerarse infinita, con la tecnología actualmente disponible hoy, sólo somos capaces de utilizar una ínfima parte de la misma. Es en este sentido en el que puede decirse que la energía es escasa y, por lo tanto, un bien económico.

50 Ibídem p. 17.

51 Ibídem p. 18. Para la discusión sobre los orígenes de la concepción dinámica de la eficiencia, veáse op. cit. pp. 14-16.

52 Es irrelevante desde el punto de vista de la economía la inmensa cantidad de energía liberada por las estrellas supernovas de otros sistemas solares a millones de años luz de la tierra, o la energía asociada a los campos gravitatorios de lejanas galaxias, de la misma manera que es irrelevante la energía contenida en la materia en forma de partículas subatómicas. 
Así pues, la energía relevante desde el punto de vista de la teoría económica, resulta ser un subconjunto —además, infinitesimal- de la cantidad total sobre la que ejerce su dominio la primera ley de la termodinámica.

Por tanto, sólo si la tecnología para extraer energía del Universo y hacerla utilizable por el hombre hubiera alcanzado el límite de sus posibilidades y no cupieran nuevos avances, podríamos decir que ésta está dada y, por lo tanto, sujeta a un problema puro de optimización - eficiencia estática.

No obstante, el hombre, al ejercer la función empresarial, crea constantemente nuevo conocimiento que amplía las fronteras de posibilidades de producción y a la vez coordina a los diversos agentes -eficiencia dinámica. Esto es así porque el ser humano, en un entorno de libertad, está constantemente alerta, dándose cuenta de nuevas oportunidades, descubriendo nuevos fines y medios y persiguiéndolos. Tal y como sostiene el profesor Huerta de Soto en su Teoría de la eficiencia dinámica,

en la vida real ni los recursos ni la tecnología están «dados», sino que pueden variar y de hecho varían continuamente como resultado de la creatividad empresarial. ${ }^{53}$

Por tanto, en ningún caso podemos afirmar categóricamente que hayamos alcanzado el límite de nuestras posibilidades, mucho menos en lo que al uso y aprovechamiento de la energía se refiere.

De este modo, si se dan las condiciones adecuadas para la eficiencia dinámica, el ser humano ejercerá su función empresarial y en el futuro - aunque no podamos predecir cuándo ni en qué forma- se desarrollarán (1) mejoras en las formas actuales de extraer la energía de la naturaleza, (2) nuevas fuentes o formas de extraer energía y (3) mejoras que permitan utilizar menos energía para lograr los mismos fines.

En este sentido, podemos afirmar junto con Julian Simon que

53 Ibídem p. 19. 
human ingenuity is the ultimate resource that, when applied to the master resource of energy, can enable people to enjoy longer, more comfortable, and more productive lives. ${ }^{54}$

En efecto, lo que olvida el posicionamiento estático es la componente de la función empresarial, la creatividad. Ver la energía como un recurso dado y limitado, cuyas fuentes están próximas a agotarse y para cuya explotación y consumo sólo interesa la búsqueda del óptimo, es la consecuencia de no considerar el principio esencial de la teoría económica de que «el hombre tiende a descubrir la información que le interesa, por lo que, si existe libertad en cuanto a la consecución de fines e intereses, estos mismos actuarán como incentivo, y harán posible que aquel que ejerce la función empresarial motivada por dicho incentivo perciba y descubra continuamente la información práctica relevante para la consecución de los fines propuestos». 55

Por eso, podemos pensar sin temor a equivocarnos - siempre y cuando se cumpla la condición de un entorno institucional que no sea hostil al ejercicio de la función empresarial-que

(...) the log-run future of our energy supply is at least as bright as that of other natural resources, though government intervention can temporarily boost prices from time to time. Finiteness is no problem here either. And the long-run impact of additional people is likely to speed the development of cheap energy supplies that are almost inexhaustible. ${ }^{56}$

\section{Energía y riqueza}

El hecho de que concedamos tanta importancia a la energía y a disponer de un entorno institucional dinámicamente eficiente proviene de su carácter de bien económico de orden superior por excelencia y, por lo tanto, de su conexión directa con la riqueza

\footnotetext{
$54 V$. Bradley y Fulmer p. xv.

$55 V$. Huerta de Soto (2005) pp. 74-75.

$56 V$. Simon (1996) p. 6.
} 
o pobreza de las personas y las sociedades en las que se desenvuelven. Pero esta conexión aun requiere de una justificación teórica.

Tenemos que demostrar, por lo tanto, en términos de teoría económica que el aumento de la energía disponible que permite un entorno institucional dinámicamente eficiente abre nuevos mercados a capas de población que aún no son consumidores marginales. ${ }^{57}$

Así, habiendo quedado demostrada la premisa de partida de que la energía está, como si dijéramos, presente o incorporada en todos los bienes de consumo como «bien de capital» ${ }^{58}$ de orden superior, si la energía se hace más abundante - porque hay nuevas formas de extraerla o porque se necesita una menor cantidad para lograr el mismo fin-, entonces por la ley praxeológica del rendimiento, la utilidad marginal de las unidades de energía extra que se «liberan» tiene que ser necesariamente menor. ${ }^{59}$

Por lo tanto, y esto es muy importante, independientemente de la diferente intensidad con la que disminuye la utilidad marginal de la energía para cada persona, se tenderá a que la valoración subjetiva de la energía sea menor. Esta menor valoración subjetiva de la energía por los agentes implica que su capacidad de intercambio, en la terminología de la teoría de determinación de los precios de Böhm-Bawerk ${ }^{60}$, aumenta. $\mathrm{Al}$ aumentar la capacidad de intercambio de los agentes, automáticamente cambian las

57 No nos referimos aquí a aquellos consumidores submarginales que se encuentran en la situación extrema que suele denominarse de pobreza energética, sino a todo el conjunto de potenciales consumidores con una demanda no satisfecha de bienes en los que la energía es un factor de producción - o sea, virtualmente todosy que dejan de producirse por el coste relativamente elevado de ésta.

58 Aunque puede resultar chocante denominar a la energía, que según hemos visto es de naturaleza abstracta e intangible, un bien de capital, debemos recordar que, como expresaba el profesor Huerta de Soto «lo que dota de naturaleza económica a un bien de capital no es su entidad física, sino el hecho de que algún actor considere que dentro de su proceso de acción le va a ser útil para alcanzar o culminar alguna etapa del mismo». V. Huerta de Soto (2009) p. 218.

59 Para una descripción de las leyes de la utilidad marginal y del rendimiento puede consultarse Mises (1949) p. 143 y ss.

60 V. Böhm-Bawerk (1889) pp. 99-142. La ley básica de determinación del precio de Eugene von Böhm-Bawerk, extracto traducido al castellano de la obra de este autor Kapital und Kapitalzins, vol II, libro III, parte B. 
parejas marginales: las antiguas dejan de serlo y aparece un nuevo juego de parejas marginales. Es decir, alguien a quien anteriormente le estaba vedado el consumo o producción de energía, pasa a formar parte del conjunto de individuos afortunados ${ }^{61}$ que toman parte del intercambio.

Como consecuencia, se amplía el mercado, dado que se producen nuevos intercambios que antes no se daban al entrar en él los productores y/o los consumidores submarginales que hasta entonces quedaban excluidos. No importa que esta ampliación del mercado de la energía se produzca en la etapa justo previa al consumo - por ejemplo por una reducción de los precios de la energía eléctrica para el suministro doméstico o del precio de la gasolina- o en fases muy alejadas del mismo - disminución del precio de las fuentes de energía primaria como el petróleo o el carbón-, dado que según la teoría económica, estos cambios se propagan a lo largo de toda la cadena causal descrita por Menger.

\section{Energía y las etapas del proceso productivo}

Terminamos este análisis de la energía desde el punto de vista praxeológico con unas acotaciones sobre el papel que ésta juega en el proceso productivo y que nos conectará directamente con la teoría del capital para afirmar que si las sociedades comparativamente más ricas son las que «tienen más tiempo acumulado en forma de bienes de capital», ${ }^{62}$ éstas son también las que tienen más energía transformada en bienes de capital. ${ }^{63}$

61 Dado que, según la teoría de la acción humana, el intercambio es un acto voluntario del ser humano para resolver una situación de insatisfacción, y que para que éste se produzca, por definición los agentes que participan valoran subjetivamente más el bien que reciben que el que ofrecen a cambio, podemos afirmar con el suficiente sustento científico, que siempre que se produzca un intercambio, ambas partes salen subjetivamente beneficiadas y, por lo tanto, cabe calificarlas de «afortunadas» (nos referímos aquí a la tercera acepción del término «afortunado» del Diccionario de la Real Academia: «Feliz, que produce felicidad o resulta de ella»).

$62 \mathrm{~V}$. Huerta de Soto (2009) p. 223. El subrayado corresponde al original.

63 No obstante, no queremos aquí decir que la energía — salvo quizás en su forma más primitiva como alimento- sea tan importante ni más que el tiempo —ahorro. Para ser capaces de producir un mecanismo capaz de generar energía aprovechable 
La teoría económica contempla la acción humana como un conjunto de etapas subjetivas que se suceden en el tiempo, en el que los estadios intermedios se encarnan subjetivamente en bienes de capital. Estos bienes de capital, a su vez, «aparecen como la conjunción acumulada de tres elementos esenciales: recursos naturales, trabajo y tiempo, todos ellos combinados a lo largo de un proceso de acción empresarial creado y emprendido por el ser humano». 64

La pregunta que nos planteamos es cómo entra en esta ecuación, formada por los tres elementos esenciales, la energía. Pues bien, considerando que para llegar a un fin determinado debemos ir quemando etapas, centrémonos ahora en el instante preciso entre una etapa y la siguiente. Independientemente de cómo de lejos nos encontremos del consumo final, nos hallaremos entonces en una situación en la que disponemos de una serie de bienes de capital, que son los necesarios para dar el salto a la siguiente etapa. ${ }^{65}$ ¿Qué nos hace falta para dar el paso, aparte del acto de voluntad humana y el tiempo? En efecto, el elemento adicional que necesitamos, a la luz de toda la exposición anterior es la energía necesaria para poder realizar la combinación de elementos que supone el acto de producción. En función de qué bien estemos produciendo, la energía que se requiera será de un tipo u otro -calor, mecánica, eléctrica, ...- pero siempre necesitaremos energía y siempre habrá una parte de la que aportemos que se quede en el producto final acabado y otra que se disipe y no sea nunca más aprovechable ${ }^{66}$.

para fines humanos, es necesario el ahorro previo. En el ejemplo de Robinson Crusoe mencionado más arriba, para poder construir el molino de agua y aprovecharse de la energía potencial del salto de agua, ha necesitado previamente reducir su consumo diario de moras para acumular suficiente cantidad de ellas para alimentarse mientras construye la noria sin necesidad de salir a recolectarlas.

${ }^{64} V$. Huerta de Soto (2009) p. 219. El término «trabajo» en esta cita se corresponde con el concepto económico de mano de obra y no en el sentido físico en el que lo hemos empleado a lo largo de este trabajo.

65 Si se tratara de una tortilla de patatas, es como si tuviéramos ya el huevo batido, las patatas fritas, la sartén con el aceite, un hornillo, la espumadera y un cocinero dispuesto a ponerse manos a la obra.

${ }^{66}$ En nuestro ejemplo culinario, la energía que necesitamos es en forma de calor, que obtenemos por intervención del hornillo, que toma el calor de la electricidad, 
Es bien cierto que la energía, a la que ya antes hemos caracterizado como un bien económico de orden superior, es realmente un factor más de producción y, por lo tanto deberíamos haberla considerado en el recuento de los bienes necesarios para acometer la etapa siguiente. No obstante, la afirmación que hacemos en este apartado, no contradice nuestra consideración de la energía como el factor de producción por excelencia, antes bien, la refuerza al darnos cuenta del hecho de que ésta debe estar siempre presente, al igual que la voluntad humana - aunque evidentemente a un nivel inferior - para que podamos cubrir cada etapa.

Siendo esto así, en cada paso estamos «consumiendo» energía, por lo que podríamos decir justificadamente que en cada bien de capital tenemos acumulada una cantidad de tiempo y de energía transformada. Y por lo tanto, podemos afirmar que las sociedades más prósperas son aquellas que han sido capaces de ahorrar y, a la vez, de convertir en útil una mayor proporción de la energía que han tenido disponible a lo largo de los siglos.

\section{$\mathrm{V}$ \\ CONCLUSIONES}

Las principales conclusiones del análisis realizado en este trabajo son las siguientes:

1. La energía es un concepto científico relativamente moderno y aún en discusión, aunque para su análisis económico es suficiente su definición como «capacidad para realizar un trabajo» y la validez a nivel macroscópico de las dos leyes fundamentales de la termodinámica: que la energía del universo es constante y que en toda transformación de energía de un tipo a otro hay una parte que se pierde para su conversión en trabajo útil.

de la reacción química que se produce en la combustión del gas, o del campo electromagnético que genera por inducción. Parte de la energía se pierde para siempre en forma de calor, parte se desaprovecha en forma de vapor de agua y otra la mantenemos para nosotros en forma de calorías alimentarias. 
2. La utilización intensiva y extensiva de la energía y las tecnologías asociadas por parte del ser humano es relativamente reciente, apenas 200 años, pero su utilización y el perfeccionamiento de las técnicas ha propiciado un avance sin precedente en los niveles de desarrollo económico y humano.

3. La energía, en tanto que es un medio para realizar un trabajo que le es útil al ser humano para la consecución de sus fines y que, como tal, es necesaria para la producción de cualquier bien, sea de capital o de consumo, puede ser considerada como el factor de producción o el bien económico de orden superior por excelencia.

4. Pese a la vigencia de las leyes de la termodinámica, no se puede considerar en ningún caso que la energía disponible para conseguir los fines de las personas esté dada y, por lo tanto, el problema económico sea el de optimización —eficiencia estática. Con el conocimiento y la tecnología actual sólo somos capaces de aprovechar una ínfima parte de la energía que hay en nuestro entorno. Seremos capaces de ampliar nuestras capacidades para extraer energía de la naturaleza de manera sostenible - y podemos considerar que no hay límite- en tanto en cuanto se den las condiciones para que la función empresarial actúe libremente y se logre así la máxima eficiencia dinámica.

5. El aumento de la energía disponible que permite un entorno institucional dinámicamente eficiente abre nuevos mercados a capas de población que aún no son consumidores marginales. Por lo tanto existe una conexión directa entre la abundancia de energía utilizable por un hombre o una sociedad y su riqueza.

6. Si las sociedades comparativamente más ricas son las que han acumulado una mayor cantidad de ahorro durante más tiempo en forma de bienes de capital, éstas necesariamente serán las que tienen más energía transformada en trabajo útil. Por lo tanto, las sociedades más prósperas son aquellas que han sido capaces de ahorrar $\mathrm{y}$, a la vez, de convertir en útil una mayor proporción de la energía que han tenido disponible a lo largo de los siglos

Tal es la importancia de la energía que no es de extrañar que gobernantes y burócratas crean que controlando la energía pueden 
alcanzar mejor sus fines económicos y políticos, dado que les es útil para controlar virtualmente a la totalidad del resto de factores de producción. Únicamente existe un bien económico -en este caso de intercambio-superior a la energía en este aspecto: el dinero. ${ }^{67}$

Seguramente por este motivo, el de la energía, junto con el financiero, es probablemente uno de los sectores más intervenidos de la economía en todos los países del mundo - sin contar seguridad y defensa donde el estado es monopolista. Y es por ello que la cuestión energética, por motivos diversos pero en gran medida ideológicos, está en la agenda política y económica, siendo varios los temas que preocupan a nuestros políticos: mix energético, liberalización del mercado, suministro y abastecimiento, ahorro energético, emisiones de gases de efecto invernadero, etc.

Debido a lo anteriormente expuesto, es más preciso hoy que nunca, cuando nuestra economía depende casi en su totalidad de la energía, poner de manifiesto y demostrar científicamente que la intervención masiva del estado en las diferentes etapas de la cadena de valor de los sectores energéticos -electricidad, gas, combustibles para el transporte- acaba por distorsionarlo, bloquea la función empresarial - que hemos visto es condición sine qua non para lograr la eficiencia dinámica que nos proveerá de energía en el futuro-y, además, sólo consigue producir efectos imprevistos e indeseados. ${ }^{68}$

67 Aun cuando el uso controlado del fuego puede que sea anterior al uso generalizado de algún bien como medio de intercambio, probablemente los seres humanos comenzaron a realizar intercambios con anterioridad. Además, fue gracias al dinero que pudieron multiplicarse los intercambios y con ellos el comercio y la civilización que, posteriormente dio lugar al desarrollo tecnológico energético. Es por eso que consideramos al dinero un factor mucho más poderoso.

68 A este respecto, podemos citar a Mises a cuenta de una de las intervenciones más conspicuas, la de los precios: «Si es cierto que los precios son consecuencia del libre funcionamiento del mercado, no pueden ser libremente manipulados por el gobierno. La intervención gubernamental es justamente un dato nuevo cuyas consecuencias el propio funcionamiento del mercado determinará, de modo que al final no tienen por qué producirse los resultados que el poder público buscaba. Las consecuencias últimas, incluso desde el punto de vista del gobernante, pueden resultar menos deseables que la situación que él mismo pretendía cambiar». $V$. Mises (1949) p. 898. 
Por este motivo, es necesario poner en marcha un programa de investigación orientado a poner de relieve todos los efectos no deseados que surgen de la intervención estatal en el campo de la energía y demuestre que existen alternativas basadas en el libre ejercicio de la función empresarial que son dinámicamente eficientes $y$, en general, suponen mejores vías para poner en valor los beneficios del ahorro y la eficiencia, dejando que sean los individuos - productores y consumidores-quienes actúen según su valoración subjetiva de la energía.

\section{REFERENCIAS BIBLIOGRÁFICAS}

BARQUín, JuLIÁN (2004): Energía: técnica, economía y sociedad, Madrid, Universidad Pontificia Comillas.

BÖHM-BAWERK, EUGENE VON (1889): «La ley básica de determinación del precio", Lecturas de economía política (Ed. Jesús Huerta de Soto), Madrid, Unión Editorial, Madrid, 1986, pp. 99142.

Bradley, Robert L. y Fulmer, Richard W. (2004): Energy. The Master Resource, Dubuque, Kendall/Hunt Publishing Company.

COROMINES, JoAn (2009): Breve diccionario etimológico de la lengua castellana, Madrid, Editorial Gredos.

Covarrubias Orozco, Sebastián de (1611): Tesoro de la lengua castellana o española, Volumen 7 de Nueva biblioteca de erudición y crítica, 2. a edición, Madrid, Editorial Castalia, 1995.

DiAmOND, JARED (1997): Guns, Germs and Steel: The Fates of Human Societies, Nueva York, Ed. W.W. Norton.

Huerta De Soto, Jesús (2009): Dinero, crédito bancario y ciclos económicos, 4. ${ }^{\text {a }}$ edición, Madrid, Unión Editorial.

- (2005): Socialismo, cálculo económico y función empresarial, 3. ${ }^{a}$ edición, Madrid, Unión Editorial.

- (2004): «La teoría de la eficiencia dinámica», Procesos de Mercado: revista Europea de Economía Política, vol. I, n. ${ }^{\circ} 1$, Primavera 2004, pp. 11-71.

- (2000): La Escuela Austriaca. Mercado y creatividad empresarial, Madrid, Ed. Síntesis. 
Jevons, W. Stanley (1866): The Coal Question. An Inquiry Concerning the Progress of the Nation and the Probable Exhaustion of our Coal Mines, 2. edición, Londres, MacMillan and Co.

Menger, CARL (1871): Principios de economía politica, 2. Edición, Madrid, Unión Editorial, 1997.

MirowsKi, PhiLip (1989): More Heat than Light. Economics as Social Physics, Physics as Nature's Economics, Cambridge, Cambridge University Press.

Mises, Ludwig Von (1949): La acción humana. Tratado de Economía, 10. ${ }^{\text {a }}$ Edición, Madrid, Unión Editorial, 2011.

Simon, Julian L. (1996): The Ultimate Resource 2, Princeton, Princeton University Press.

Vega, Lope de (1632): La Dorotea, Madrid y Los Ángeles, Edición conjunta de University of California Press y Editorial Castalia, 1968.

WEINER, JACK (2003): «El indispensable factótum Sebastián de Covarrubias Horozco (1539-1613): pedagogo, cortesano y administrador», Artifara, n. 2, (enero - junio 2003), sezione Addenda. 